Research Paper

\title{
The Association between GWAS-identified BARDI Gene SNPs and Neuroblastoma Susceptibility in a Southern Chinese Population
}

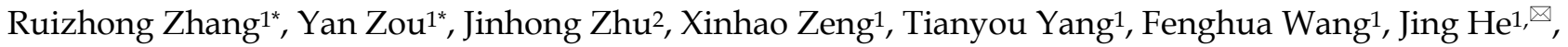 \\ Huimin Xia ${ }^{1, \bigotimes}$ \\ 1. Department of Pediatric Surgery, Guangzhou Women and Children's Medical Center, Guangzhou Medical University, Guangzhou 510623, Guangdong, \\ China; \\ 2. Molecular Epidemiology Laboratory and Department of Laboratory Medicine, Harbin Medical University Cancer Hospital, Harbin 150040, Heilongjiang, \\ China. \\ * Ruizhong Zhang and Yan Zou contribute equally to this work.
}

$\triangle$ Corresponding authors: Huimin Xia, Department of Pediatric Surgery, Guangzhou Women and Children's Medical Center, Guangzhou Medical University, 9 Jinsui Road, Guangzhou 510623, Guangdong, China, Tel.: (+86-020) 38076001, Fax: (+86-020) 38076020; E-mail: xia-huimin@foxmail.com; or Jing He, Department of Pediatric Surgery, Guangzhou Women and Children's Medical Center, Guangzhou Medical University, 9 Jinsui Road, Guangzhou 510623, Guangdong, China, Tel./Fax: (+86-020) 38076560, E-mail: hejing198374@gmail.com.

(0) Ivyspring International Publisher. Reproduction is permitted for personal, noncommercial use, provided that the article is in whole, unmodified, and properly cited. See http://ivyspring.com/terms for terms and conditions.

Received: 2015.08.02; Accepted: 2016.01.05; Published: 2016.02.03

\begin{abstract}
A previous genome-wide association study (GWAS) has found that some common variations in the BARDI gene were associated with neuroblastoma susceptibility especially for high-risk subjects, and the associations have been validated in Caucasians and African-Americans. However, the associations between BARDI gene polymorphisms and neuroblastoma susceptibility have not been studied among Asians, not to mention Chinese subjects. In the present study, we investigated the association of three BARDI polymorphisms (rs7585356 G>A, rs6435862 T>G and rs3768716 $A>G$ ) with neuroblastoma susceptibility in 201 neuroblastoma patients and 531 controls using TaqMan methodology. Overall, none of these polymorphisms was significantly associated with neuroblastoma susceptibility. However, stratified analysis showed a more profound association between neuroblastoma risk and rs6435862 TG/GG variant genotypes among older children (adjusted $\mathrm{OR}=1.55,95 \% \mathrm{Cl}=1.04-2.31$ ), and children with adrenal gland-originated disease (adjusted $\mathrm{OR}=2.94,95 \% \mathrm{Cl}=1.40-6.18$ ), or with ISSN clinical stages III+IV disease (adjusted $\mathrm{OR}=1.75$, $95 \% \mathrm{Cl}=1.09-2.84)$. Similar results were observed for the variant genotypes of $r s 3768716 \mathrm{~A}>\mathrm{G}$ polymorphism among these three subgroups. Our results suggest that the BARDI rs6435862 T>G and rs3768716 $A>G$ polymorphisms may contribute to increased susceptibility to neuroblastoma, especially for the subjects at age $\geq 12$ months, with adrenal gland-originated or with late clinical stage neuroblastoma. These findings need further validation by prospective studies with larger sample size with subjects enrolled from multicenter, involving different ethnicities.
\end{abstract}

Key words: BARD1; GWAS; polymorphism; neuroblastoma; susceptibility.

\section{Introduction}

Neuroblastoma has been recognized as one of the most commonly diagnosed extracranial solid tumor in infancy, which constitute about $7-10 \%$ of all childhood cancers. It is the third leading cause of cancer-related death in children [1]. The peak incidence of neuroblastoma is in children, and the median age at diagnosis is around 17 months [2]. It may arise anywhere of the sympathetic nervous system, and mainly arise within the abdomen and adrenal medulla [3]. The incidence rate of neuroblastoma is about 1 in 7000 live newborns worldwide, and nearly 700 new cases occur per year in the United States [4]. It is 
also one of the most common solid tumors in the Chinese infants, with an incidence rate of approximately 7.7 per million [5]. The majority of neuroblastomas are sporadically, and only about $1 \%$ of neuroblastoma patients have a family history [6]. So far, the etiology of neuroblastoma is not well understood [7]. Case-control studies and family studies play important roles in discovering genetic component of the neuroblastoma susceptibility [8]. For example, Han et al. [9] performed a case-control study among Chinese with 203 neuroblastoma patients and 411 controls. They found significant association of FAS -1377 G/A and FASL -844 T/C polymorphisms with neuroblastoma susceptibility.

Genome-wide association studies (GWASs) have proven to be a powerful and hypothesis-free method to discover genes that confer susceptibility to complex diseases including cancers [10]. To date, five GWASs on the neuroblastoma have been performed, mainly in European descents, and several neuroblastoma susceptibility related loci have been identified [11-15]. The first GWAS performed by Maris et al. included 1032 neuroblastoma cases and 2043 controls at the discovery stage, and 720 neuroblastoma cases and 2128 controls in the validation stage [11]. They found that three single nucleotide polymorphisms (SNPs) located on chromosome 6p22 were significantly associated with neuroblastoma susceptibility. When the analysis was restricted to only 397 high-risk neuroblastoma cases and 2043 controls [11], they observed new significant association between neuroblastoma susceptibility and six SNPs at $2 \mathrm{q} 35$ within the BRCA1 associated RING domain 1 (BARD1) locus. Of them, the rs6435862 $\mathrm{T}>\mathrm{G}$ and rs3768716 $\mathrm{A}>\mathrm{G}$ are the two most significant SNPs. The association between GWAS-identified polymorphisms in the BARD1 gene and neuroblastoma susceptibility has been validated in the African-Americans [16] as well as Italians [17], but not in Asians. With this in mind, we carried out the current hospital-based case-control study with a total of 201 neuroblastoma patients and 531 cancer-free controls to explore the association between three GWAS-identified BARD1 gene polymorphisms (rs7585356 G>A, rs6435862 T>G and rs3768716 A>G) and neuroblastoma susceptibility in a Southern Chinese population.

\section{Materials and methods}

\section{Study subjects}

We enrolled a total of 201 neuroblastoma cases as well as 531 cancer-free controls in this hospital-based case-control study as we described previously [18]. All the neuroblastoma cases were newly diagnosed and histopathologically confirmed individuals and recruited from the Guangzhou Women and Children's Medical Center. The cancer-free controls were randomly selected from children receiving a routine physical examination in the same hospital and matched to cases on age and gender (frequency matching). Both of the cases and controls were ethnic Chinese Han subjects. Exclusion criteria were as follows: other types of cancer, secondary/recurrent malignancies, and receipt of chemotherapy or radiotherapy before recruitment. At recruitment, information on each subject (e.g., age, gender and personal medical histories) was collected by structured questionnaire or medical records. This study was approved by the Institutional Review Board of Guangzhou Women and Children's Medical Center. Written informed consent was obtained from all participants or the children's guardians.

\section{Polymorphism analysis}

Genomic DNA was mainly extracted from $2 \mathrm{~mL}$ blood sample using the TIANamp Blood DNA Kit (TianGen Biotech Co. Ltd., Beijing, China) according to the manufacturer's instructions. DNA samples were prepared as we described previously $[19,20]$. Briefly, all the DNA samples were diluted to a concentration of $10 \mathrm{ng} / \mu \mathrm{L}$ and loaded in the 96-well plates. Genotyping for the three GWAS-identified BARD1 SNPs (rs7585356 G>A, rs6435862 T>G, and rs3768716 $A>G$ ) [12] was performed in the 384-well plate using Taqman method as published previously [19]. As shown in Supplemental Table 1, these three SNPs can also capture an additional of 10 polymorphisms as predicted by SNPinfo software (http://snpinfo.niehs.nih.gov/snpinfo/snpfunc.htm) . Moreover, $10 \%$ of samples were selected randomly for repeat assay, and the results were $100 \%$ concordant.

\section{Statistical analysis}

Distributions of demographic variables and genotypes between cases and controls were compared by $\chi^{2}$ test. Goodness-of-fit $\chi^{2}$ test was performed to detect deviation from Hardy-Weinberg equilibrium in controls. Odds ratios (ORs) and 95\% confidence intervals (CIs) adjusted for age and gender were used to assess the strength of associations between selected polymorphisms and neuroblastoma susceptibility by using unconditional multivariate logistic regression analysis. All statistical analyses were performed using SAS software (version 9.1; SAS Institute, Cary, NC), with a significance level of 0.05. All tests were two-sided. 


\section{Results}

\section{Population characteristics}

The distributions of the demographic characteristics of the neuroblastoma cases and controls were summarized in Supplemental Table 2. The current study included 201 neuroblastoma patients and 531 age- and gender-matched cancer-free controls. No statistical significant differences were observed in the distributions of age $(P=0.788)$ and gender $(P=0.452)$ between cases and controls. According to INSS criteria [21], $50(24.88 \%), 54(26.87 \%), 34(16.92 \%), 49$ $(24.38 \%)$ and 7 (3.48\%) patients had clinical stage I, II, III, IV and 4s neuroblastoma, respectively. In term of tumor site, the neuroblastomas mainly occurred in adrenal glands $(\mathrm{N}=30,14.93 \%)$, retroperitoneal regions $(\mathrm{N}=50,24.86 \%)$, and mediastinum $(\mathrm{N}=80$, $39.80 \%)$.

\section{Association between BARDI SNPs and neu- roblastoma susceptibility}

The genotype frequencies of the three selected SNPs and their associations with neuroblastoma sus- ceptibility were shown in Table $\mathbf{1}$. We observed that frequency distributions of all of the BARD1 polymorphisms were consistent with the Hardy-Weinberg equilibrium $(P=0.948$ for $r 57585356 \mathrm{G}>\mathrm{A}, P=0.205$ for rs6435862 $\mathrm{T}>\mathrm{G}$, and $P=0.415$ for rs3768716 $\mathrm{A}>\mathrm{G}$ polymorphism) in control subjects. We failed to observe any significant association between the rs7585356 G>A polymorphism and neuroblastoma susceptibility (AG vs. GG: adjusted OR=0.91, 95\% CI=0.64-1.27; AA vs. GG: adjusted $\mathrm{OR}=0.61,95 \% \mathrm{CI}=0.33-1.13$; AG/AA vs. GG: adjusted $\mathrm{OR}=0.85,95 \% \mathrm{CI}=0.61-1.17$ and GG/AG vs. AA: adjusted OR=0.64, 95\% CI=0.35-1.16). As to the rs6435862 $\mathrm{T}>\mathrm{G}$ polymorphism, we found a borderline significant increase in the neuroblastoma risk only for the rs6435862 TG carriers (adjusted $\mathrm{OR}=1.40,95 \% \mathrm{CI}=0.98-2.00, P=0.067)$ when compared to the TT carriers. A similar trend toward increased risk were observed for the rs3768716 heterozygotes (AG vs. AA: adjusted $\mathrm{OR}=1.40,95 \% \mathrm{CI}=0.98-2.00$, $P=0.076)$. We did not find any significant association for the risk genotypes.

Table 1. Logistic regression analysis of associations between BARDI polymorphisms and neuroblastoma susceptibility.

\begin{tabular}{|c|c|c|c|c|c|c|c|}
\hline Genotype & Cases $(\mathrm{N}=201)$ & Controls $(\mathrm{N}=531)$ & $P$ a & Crude OR (95\% CI) & $P$ & Adjusted OR $(95 \% \mathrm{CI}) \mathrm{b}$ & $P^{\mathrm{b}}$ \\
\hline \multicolumn{8}{|c|}{ rs7585356 (HWE=0.948) } \\
\hline GG & $97(48.26)$ & $235(44.26)$ & & 1.00 & & 1.00 & \\
\hline AG & $89(44.28)$ & $237(44.63)$ & & $0.91(0.65-1.28)$ & 0.585 & $0.91(0.64-1.27)$ & 0.567 \\
\hline AA & $15(7.46)$ & $59(11.11)$ & & $0.62(0.33-1.14)$ & 0.122 & $0.61(0.33-1.13)$ & 0.114 \\
\hline Additive & & & 0.295 & $0.84(0.65-1.08)$ & 0.160 & $0.83(0.65-1.07)$ & 0.148 \\
\hline Dominant & 104 (51.74) & $296(55.74)$ & 0.332 & $0.85(0.62-1.18)$ & 0.332 & $0.85(0.61-1.17)$ & 0.315 \\
\hline Recessive & $186(92.54)$ & $472(88.89)$ & 0.144 & $0.65(0.36-1.17)$ & 0.147 & $0.64(0.35-1.16)$ & 0.139 \\
\hline \multicolumn{8}{|c|}{ rs6435862 (HWE=0.205) } \\
\hline TT & $132(65.67)$ & $381(71.75)$ & & 1.00 & & 1.00 & \\
\hline TG & $64(31.84)$ & $133(25.05)$ & & $1.40(0.97-1.99)$ & 0.072 & $1.40(0.98-2.00)$ & 0.067 \\
\hline GG & $5(2.49)$ & $17(3.20)$ & & $0.85(0.31-2.35)$ & 0.752 & $0.84(0.31-2.34)$ & 0.745 \\
\hline Additive & & & 0.172 & $1.20(0.89-1.62)$ & 0.222 & $1.21(0.90-1.63)$ & 0.216 \\
\hline Dominant & $69(34.33)$ & $150(28.25)$ & 0.109 & $1.33(0.94-1.88)$ & 0.109 & $1.34(0.94-1.89)$ & 0.104 \\
\hline Recessive & $196(97.51)$ & $514(96.80)$ & 0.614 & $0.77(0.28-2.12)$ & 0.615 & $0.77(0.28-2.10)$ & 0.604 \\
\hline \multicolumn{8}{|c|}{ rs3768716 (HWE=0.415) } \\
\hline AA & $125(62.19)$ & 364 (68.55) & & 1.00 & & 1.00 & \\
\hline AG & $69(34.33)$ & $148(27.87)$ & & $1.36(0.96-1.93)$ & 0.087 & $1.38(0.97-1.96)$ & 0.076 \\
\hline GG & $7(3.48)$ & $19(3.58)$ & & $1.07(0.44-2.61)$ & 0.877 & $1.06(0.43-2.57)$ & 0.907 \\
\hline Additive & & & 0.230 & $1.22(0.92-1.63)$ & 0.170 & $1.23(0.92-1.64)$ & 0.163 \\
\hline Dominant & $76(37.81)$ & 167 (31.45) & 0.103 & $1.33(0.94-1.86)$ & 0.103 & $1.34(0.95-1.88)$ & 0.094 \\
\hline Recessive & 194 (96.52) & 512 (96.42) & 0.950 & $0.97(0.40-2.35)$ & 0.951 & $0.96(0.40-2.31)$ & 0.918 \\
\hline \multicolumn{8}{|c|}{ Combined effect of risk genotypes } \\
\hline 0 & $49(24.38)$ & $129(24.29)$ & $0.171^{c}$ & 1.00 & & 1.00 & \\
\hline 1 & $77(38.31)$ & $244(45.95)$ & & $0.83(0.55-1.26)$ & 0.384 & $0.83(0.54-1.25)$ & 0.369 \\
\hline 2 & $53(26.37)$ & 105 (19.77) & & $1.33(0.83-2.12)$ & 0.232 & $1.34(0.84-2.13)$ & 0.225 \\
\hline 3 & $22(10.95)$ & $53(9.98)$ & & $1.09(0.60-1.98)$ & 0.770 & $1.10(0.60-1.99)$ & 0.763 \\
\hline $1-3$ & $152(75.62)$ & $402(75.71)$ & 0.981 & $1.00(0.68-1.45)$ & 0.981 & $0.99(0.68-1.45)$ & 0.973 \\
\hline
\end{tabular}

a $\chi^{2}$ test for genotype distributions between neuroblastoma patients and controls.

$\mathrm{b}$ Adjusted for age and gender.

c Additive models. 
Table 2. Stratification analysis for association between BARDI genotypes and neuroblastoma susceptibility.

\begin{tabular}{|c|c|c|c|c|c|c|c|c|c|c|c|c|}
\hline \multirow[t]{2}{*}{ Variables } & \multicolumn{2}{|c|}{$\begin{array}{l}\text { rs7585356 } \\
\text { (cases/controls) }\end{array}$} & \multirow[t]{2}{*}{$\begin{array}{l}\text { Adjusted OR a } \\
(95 \% \mathrm{CI})\end{array}$} & \multirow[t]{2}{*}{$P$ a } & \multicolumn{2}{|c|}{$\begin{array}{l}\text { rs6435862 (cas- } \\
\text { es/controls) }\end{array}$} & \multirow[t]{2}{*}{$\begin{array}{l}\text { Adjusted OR a } \\
(95 \% \mathrm{CI})\end{array}$} & \multirow[t]{2}{*}{$P$ a } & \multicolumn{2}{|c|}{$\begin{array}{l}\text { rs3768716 (cas- } \\
\text { es/controls) }\end{array}$} & \multirow[t]{2}{*}{$\begin{array}{l}\text { Adjusted OR a } \\
(95 \% \mathrm{CI})\end{array}$} & \multirow[t]{2}{*}{$P$ a } \\
\hline & GG & AG/AA & & & TT & TG/GG & & & AA & AG/GG & & \\
\hline \multicolumn{13}{|l|}{ Age, month } \\
\hline$<12$ & $22 / 67$ & $32 / 78$ & $\begin{array}{l}1.23 \\
(0.65-2.32)\end{array}$ & 0.529 & $41 / 105$ & $13 / 40$ & $\begin{array}{l}0.85 \\
(0.41-1.77)\end{array}$ & 0.670 & $38 / 98$ & $16 / 47$ & $\begin{array}{l}0.90 \\
(0.45-1.78)\end{array}$ & 0.756 \\
\hline$\geq 12$ & $75 / 168$ & $72 / 218$ & $\begin{array}{l}0.74 \\
(0.50-1.08)\end{array}$ & 0.119 & $91 / 276$ & $56 / 110$ & $\begin{array}{l}1.55 \\
(1.04-2.31)\end{array}$ & 0.032 & $87 / 266$ & $60 / 120$ & $\begin{array}{l}1.54 \\
(1.04-2.28)\end{array}$ & 0.032 \\
\hline \multicolumn{13}{|l|}{ Gender } \\
\hline Females & $48 / 102$ & $34 / 131$ & $\begin{array}{l}0.55 \\
(0.33-0.92)\end{array}$ & 0.022 & $53 / 160$ & $29 / 73$ & $\begin{array}{l}1.20 \\
(0.70-2.04)\end{array}$ & 0.504 & $49 / 150$ & $33 / 83$ & $\begin{array}{l}1.22 \\
(0.73-2.04)\end{array}$ & 0.458 \\
\hline Males & $49 / 133$ & $70 / 165$ & $\begin{array}{l}1.15 \\
(0.75-1.77)\end{array}$ & 0.522 & $79 / 221$ & $40 / 77$ & $\begin{array}{l}1.45 \\
(0.92-2.30)\end{array}$ & 0.113 & $76 / 214$ & $43 / 84$ & $\begin{array}{l}1.44 \\
(0.92-2.27)\end{array}$ & 0.112 \\
\hline \multicolumn{13}{|l|}{ Sites of origin } \\
\hline Adrenal gland & $15 / 235$ & $15 / 296$ & $\begin{array}{l}0.80 \\
(0.38-1.66)\end{array}$ & 0.543 & $14 / 381$ & $16 / 150$ & $\begin{array}{l}2.94 \\
(1.40-6.18)\end{array}$ & 0.005 & $14 / 364$ & $16 / 167$ & $\begin{array}{l}2.55 \\
(1.21-5.37)\end{array}$ & 0.014 \\
\hline Retroperitoneal & $21 / 235$ & $29 / 296$ & $\begin{array}{l}1.09 \\
(0.61-1.97)\end{array}$ & 0.767 & $36 / 381$ & $14 / 150$ & $\begin{array}{l}1.00 \\
(0.52-1.91)\end{array}$ & 0.996 & $36 / 364$ & $14 / 167$ & $\begin{array}{l}0.87 \\
(0.45-1.65)\end{array}$ & 0.660 \\
\hline Mediastinum & $43 / 235$ & $37 / 296$ & $\begin{array}{l}0.68 \\
(0.43-1.10)\end{array}$ & 0.114 & $54 / 381$ & $26 / 150$ & $\begin{array}{l}1.22 \\
(0.73-2.02)\end{array}$ & 0.450 & $51 / 364$ & $29 / 167$ & $\begin{array}{l}1.23 \\
(0.75-2.01)\end{array}$ & 0.410 \\
\hline Others & $10 / 235$ & $7 / 296$ & $\begin{array}{l}0.56 \\
(0.21-1.49)\end{array}$ & 0.242 & $15 / 381$ & $2 / 150$ & $\begin{array}{l}0.35 \\
(0.08-1.57)\end{array}$ & 0.172 & $13 / 364$ & $4 / 167$ & $\begin{array}{l}0.70 \\
(0.22-2.18)\end{array}$ & 0.534 \\
\hline \multicolumn{13}{|l|}{ Clinical stages } \\
\hline $\mathrm{I}+\mathrm{II}+4 \mathrm{~s}$ & $52 / 235$ & $59 / 296$ & $\begin{array}{l}0.90 \\
(0.60-1.36)\end{array}$ & 0.621 & $76 / 381$ & $35 / 150$ & $\begin{array}{l}1.18 \\
(0.75-1.83)\end{array}$ & 0.477 & $73 / 364$ & $38 / 167$ & $\begin{array}{l}1.13 \\
(0.73-1.75)\end{array}$ & 0.580 \\
\hline III+IV & $41 / 235$ & $42 / 296$ & $\begin{array}{l}0.80 \\
(0.50-1.27)\end{array}$ & 0.347 & $49 / 381$ & $34 / 150$ & $\begin{array}{l}1.75 \\
(1.09-2.84)\end{array}$ & 0.022 & $47 / 364$ & $36 / 167$ & $\begin{array}{l}1.69 \\
(1.05-2.72)\end{array}$ & 0.031 \\
\hline
\end{tabular}

a Adjusted for age and gender.

\section{Stratified analysis of BARDI polymorphisms and neuroblastoma susceptibility}

We performed stratified analyses by age, gender, sites of origin, and clinical stages to evaluate the effects of variant genotypes on the risk of neuroblastoma (Table 2). Among the girls, carrier of rs7585356 AG or AA genotype had an odds ratio of 0.55 (adjusted OR=0.55, 95\% CI=0.33-0.92) for developing neuroblastoma, compared with carriers of GG genotype, suggesting a protective effect of rs7585356 on girls. Moreover, a comparison of homozygotes and heterozygotes versus wild-types indicated that the rs6435862 $\mathrm{T}>\mathrm{G}$ polymorphism increased the risk of neuroblastoma among the kids older than 12 months (adjusted OR=1.55, 95\% CI=1.04-2.31), with tumor in adrenal gland (adjusted OR=2.94, 95\% CI=1.40-6.18), and with clinical stages III+IV disease (adjusted $\mathrm{OR}=1.75,95 \% \mathrm{CI}=1.09-2.84$ ), when compared to the TT genotype, we observed the TG/GG carriers have an increased neuroblastoma susceptibility. Similar risk effects were observed for the rs3768716 $A>G$ polymorphism among children older than 12 months (adjusted $\mathrm{OR}=1.54,95 \% \mathrm{CI}=1.04-2.28$ ), with tumor in adrenal gland (adjusted $\mathrm{OR}=2.55,95 \% \mathrm{CI}=1.21-5.37$ ), and with clinical stages III+IV disease (adjusted $\mathrm{OR}=1.69,95 \% \mathrm{CI}=1.05-2.72$ ).

\section{Discussion}

In the current hospital-based case-control study, we explored the association of three BARD1 gene polymorphisms with neuroblastoma susceptibility in 201 patients and 531 cancer-free controls. To the best of our knowledge, this is the first investigation to validate GWAS-identified SNPs at 2 q35 within the $B A R D 1$ gene in Southern Chinese population. We found the frequency of the TG/GG genotypes of the rs6435862 $\mathrm{T}>\mathrm{G}$ polymorphism and the AG/GG genotypes of the rs3768716 $A>G$ polymorphism were significantly higher than that of their respective wide-type genotypes in older subjects, and those with disease originated from adrenal gland or late clinical stage neuroblastoma. The results from the current study suggest that rs6435862 $T>G$ and $r s 3768716 \mathrm{~A}>\mathrm{G}$ polymorphisms were significantly associated with neuroblastoma susceptibility for subjects with late clinical stage neuroblastoma, which were consistent with the findings from previous GWAS studies [12].

The BARD1 gene is located at chromosome $2 \mathrm{q} 35$, containing 13 exons. This gene encodes a protein that can interact with the $\mathrm{N}$-terminal region of BRCA1 both in vivo and in vitro [22]. The BARD1 gene has been recognized as a classically tumor suppressor for the following reasons: 1 ) it directly interacts with the BRCA1 through their respective RING domains; 2) it plays an important role in double-strand break repair and ubiquitination; 3 ) it serves as a mediator in the process of apoptosis by binding to and stabilizing p53 [23]; 4) it also plays roles in the regulation of cell growth, including the products of dominant protooncogenes and tumor suppressor genes [24]. Somatically acquired missense BARD1 mutations were 
observed in the breast and ovarian cancer patients [25]. Polymorphisms of BARD1 such as the Cys557Ser may contribute to the susceptibility of breast cancer [26-28]. However, a meta-analysis collecting a total of 14 studies with 11870 cases and 7687 controls did not validate the significant association between Cys557Ser mutation and breast cancer risk [29].

SNPs may change the encoding amino acids (non-synonymous SNPs), may be silent (synonymous SNPs), or may occur in the non-coding regions. The non-synonymous SNPs could affect the function and expression levels of genes and consequentially result in disease [30]. There are at least 4941 SNPs in the BARD1 gene region (http://www.ncbi.nlm.nih.gov/ projects/SNP). Among all the identified BARD1 SNPs, six common polymorphisms have been found to be associated with neuroblastoma susceptibility in a previous GWAS [12]. In this study, Capasso et al. only focused on high-risk neuroblastoma patients which were enrolled in their first neuroblastoma GWAS [11], a total of 397 cases and 2043 controls were included in the discover stage, and six SNPs at $2 q 35$ within the BARD1 were found to be significantly associated with increased neuroblastoma susceptibility. A total of 189 cases and 1178 controls were used to further validated the significant SNPs. Of them, the rs6435862 $\mathrm{T}>\mathrm{G}$ and rs3768716 $\mathrm{A}>\mathrm{G}$ polymorphism were the most significant for high-risk neuroblastoma patients, with allelic OR of 1.68 for each polymorphism.

In the first replication study carried out among African-Americans, comprising 390 neuroblastoma patients, Latorre et al. [16] found that all of the included SNPs were associated with increased neuroblastoma risk with one exception that they failed to confirm the association of the first GWAS-identified SNPs within the FLJ22536 gene with neuroblastoma susceptibility. In another replication study in Italians with 370 neuroblastoma cases and 809 controls, Capasso et al. [17] proved that the BARD1 SNPs were associated with neuroblastoma susceptibility, and the association was more prominent for high-risk neuroblastoma patients. In the current study, we chose the two most significant SNPs (rs6435862 T>G and rs3768716 A>G) as well as the one located in the $3^{\prime}$ UTR region (rs7585356 G>A). We failed to find the significant associations between the selected SNPs and neuroblastoma susceptibility for overall subjects. Interestingly, in the stratified analysis by clinical stages, we found subjects carrying the rs6435862 TG/GG or rs3768716 AG/GG genotypes have a significantly increased risk of developing neuroblastoma among the ISSN clinical stages III/IV neuroblastoma patients. The reason we failed to validate the results from the studies conducted among Afri-
can-Americans and Italians may be ascribed to the ethnicity difference. For example, the minor allele frequency (MAF) of the rs6435862 $\mathrm{T}>\mathrm{G}$ was 0.18 for our neuroblastoma cases and 0.16 for the cancer-free controls, 0.34 for African-American cases and 0.26 for and controls, and 0.43 for Italian cases and 0.26 for controls. As to the rs3768716 A $>$ G polymorphism, the MAF for the cases and controls in the current study was 0.21 and $0.18,0.10$ and 0.07 for African-American cases and controls, and 0.35 and 0.23 for Italian cases and controls, respectively. Given the possible differences in the MAF and pattern of linkage disequilibrium among Asians, African-Americans and Caucasians, the effects of the studied genetic susceptibility to neuroblastoma may vary, which may partially explain the failure to validate the significant results from African-Americans and Italians. Besides, the first GWAS also did not detect the association of the $B A R D 1$ polymorphisms with neuroblastoma risk in all subjects [11]. We speculate that these SNPs within the BARD1 gene may have mild contribution to the development of neuroblastoma. The relatively small sample size of this study might have limited statistical power to detect such mild effect of studied SNPs.

This is the first validation study for the association between BARD1 gene polymorphisms and neuroblastoma susceptibility in Southern Chinese children. There were several potential limitations should be addressed in the present study. First, only 201 patients were included, the relatively small sample size may have reduced the statistical power of the study. Second, we only included three polymorphisms in the BARD1 gene, more polymorphisms especially the potentially functional SNPs not contained in GWASs remain to be replicated. Finally, this study was restricted to Chinese Han ethnicity subjects from Southern China, and the results should be extrapolated to other ethnic groups cautiously.

In conclusion, in the current study, we found a significant association of the BARD1 gene rs6435862 $\mathrm{T}>\mathrm{G}$ and rs3768716 $\mathrm{A}>\mathrm{G}$ polymorphisms with an increased neuroblastoma susceptibility for older children, children with adrenal gland-originated or late clinical stage neuroblastoma subjects in a Chinese Han population. Further prospective studies with larger sample sizes including different ethnic populations and further functional studies are required to validate our results.

\section{Abbreviations}

GWAS, genome-wide association study; SNP, single nucleotide polymorphism; BARD1, BRCA1 associated RING domain 1; OR, odds ratio; CI, confidence interval; MAF, minor allele frequency. 


\section{Supplementary Material}

Supplemental Tables 1 and 2.

http://www.medsci.org/v13p0133s1.pdf

\section{Acknowledgement}

This work was supported by the grant of State Clinical Key Specialty Construction Project (Pediatric Surgery) 2013, (No: GJLCZD1301) and the grant of clinical medicine research and transformation center of brain injury in premature infant in Guangzhou (No: 520101-2150092). We thank Yanlu Tong and Hezhen Wang for their assistance in DNA extraction and medical histories information collection.

\section{Competing Interests}

The authors have declared that no competing interest exists.

\section{References}

1. Smith MA, Seibel NL, Altekruse SF, Ries LA, Melbert DL, O'Leary M, et al. Outcomes for children and adolescents with cancer: challenges for the twenty-first century. J Clin Oncol. 2010; 28: 2625-34

2. London WB, Castleberry RP, Matthay KK, Look AT, Seeger RC, Shimada H, et al. Evidence for an age cutoff greater than 365 days for neuroblastoma risk group stratification in the Children's Oncology Group. J Clin Oncol. 2005; 23: 6459-65.

3. Deyell RJ, Attiyeh EF. Advances in the understanding of constitutional and somatic genomic alterations in neuroblastoma. Cancer Genet. 2011; 204: $113-21$.

4. Gurney JG, Ross JA, Wall DA, Bleyer WA, Severson RK, Robison LL. Infant cancer in the U.S.: histology-specific incidence and trends, 1973 to 1992. J Pediatr Hematol Oncol. 1997; 19: 428-32.

5. Bao PP, Li K, Wu CX, Huang ZZ, Wang CF, Xiang YM, et al. [Recent incidences and trends of childhood malignant solid tumors in Shanghai, 2002-2010]. Zhonghua Er Ke Za Zhi. 2013; 51: 288-94.

6. Shojaei-Brosseau T, Chompret A, Abel A, de Vathaire F, Raquin MA, Brugieres L, et al. Genetic epidemiology of neuroblastoma: a study of 426 cases at the Institut Gustave-Roussy in France. Pediatr Blood Cancer. 2004; 42: 99-105.

7. Capasso M, Diskin SJ. Genetics and genomics of neuroblastoma. Cancer Treat Res. 2010; 155: 65-84.

8. Sridhar S, Al-Moallem B, Kamal H, Terrile M, Stallings RL. New insights into the genetics of neuroblastoma. Mol Diagn Ther. 2013; 17: 63-9.

9. Han W, Zhou Y, Zhong R, Wu C, Song R, Liu L, et al. Functional polymorphisms in FAS/FASL system increase the risk of neuroblastoma in Chinese population. PLoS One. 2013; 8: e71656.

10. Manolio TA. Genomewide association studies and assessment of the risk of disease. N Engl J Med. 2010; 363: 166-76.

11. Maris JM, Mosse YP, Bradfield JP, Hou C, Monni S, Scott RH, et al. Chromosome 6p22 locus associated with clinically aggressive neuroblastoma. N Engl J Med. 2008; 358: 2585-93.

12. Capasso M, Devoto M, Hou C, Asgharzadeh S, Glessner JT, Attiyeh EF, et al. Common variations in BARD1 influence susceptibility to high-risk neuroblastoma. Nat Genet. 2009; 41: 718-23.

13. Nguyen le B, Diskin SJ, Capasso M, Wang K, Diamond MA, Glessner J, et al. Phenotype restricted genome-wide association study using a gene-centric approach identifies three low-risk neuroblastoma susceptibility Loci. PLoS Genet. 2011; 7: e1002026.

14. Wang K, Diskin SJ, Zhang H, Attiyeh EF, Winter C, Hou C, et al. Integrative genomics identifies LMO1 as a neuroblastoma oncogene. Nature. 2011; 469: 216-20.

15. Diskin SJ, Capasso M, Schnepp RW, Cole KA, Attiyeh EF, Hou C, et al. Common variation at $6 \mathrm{q} 16$ within HACE1 and LIN28B influences susceptibility to neuroblastoma. Nat Genet. 2012; 44: 1126-30.

16. Latorre V, Diskin SJ, Diamond MA, Zhang H, Hakonarson H, Maris JM, et al. Replication of neuroblastoma SNP association at the BARD1 locus in African-Americans. Cancer Epidemiol Biomarkers Prev. 2012; 21: 658-63.

17. Capasso M, Diskin SJ, Totaro F, Longo L, De Mariano M, Russo R, et al. Replication of GWAS-identified neuroblastoma risk loci strengthens the role of BARD1 and affirms the cumulative effect of genetic variations on disease susceptibility. Carcinogenesis. 2013; 34: 605-11.

18. He J, Zhang R, Zou Y, Zhu J, Yang T, Wang F, et al. Evaluation of GWAS-identified SNPs at 6 p22 with neuroblastoma susceptibility in a Chinese population. Tumour Biol. 2015. [Epub ahead of Print]
19. He J, Qiu LX, Wang MY, Hua RX, Zhang RX, Yu HP, et al. Polymorphisms in the XPG gene and risk of gastric cancer in Chinese populations. Hum Genet. 2012; 131: 1235-44.

20. He J, Wang MY, Qiu LX, Zhu ML, Shi TY, Zhou XY, et al. Genetic variations of mTORC1 genes and risk of gastric cancer in an Eastern Chinese population. Mol Carcinog. 2013; 52 (Suppl 1): E70-9.

21. Brodeur GM, Pritchard J, Berthold F, Carlsen NL, Castel V, Castelberry RP, et al. Revisions of the international criteria for neuroblastoma diagnosis, staging, and response to treatment. J Clin Oncol. 1993; 11: 1466-77.

22. Wu LC, Wang ZW, Tsan JT, Spillman MA, Phung A, Xu XL, et al. Identification of a RING protein that can interact in vivo with the BRCA1 gene product. Nat Genet. 1996; 14: 430-40.

23. Feki A, Jefford CE, Berardi P, Wu JY, Cartier L, Krause KH, et al. BARD1 induces apoptosis by catalysing phosphorylation of p53 by DNA-damage response kinase. Oncogene. 2005; 24: 3726-36.

24. Irminger-Finger I, Jefford CE. Is there more to BARD1 than BRCA1? Nat Rev Cancer. 2006; 6: 382-91.

25. Thai TH, Du F, Tsan JT, Jin Y, Phung A, Spillman MA, et al. Mutations in the BRCA1-associated RING domain (BARD1) gene in primary breast, ovarian and uterine cancers. Hum Mol Genet. 1998; 7: 195-202.

26. Stacey SN, Sulem P, Johannsson OT, Helgason A, Gudmundsson J, Kostic JP, et al. The BARD1 Cys557Ser variant and breast cancer risk in Iceland. PLoS Med. 2006; 3: e217.

27. Karppinen SM, Barkardottir RB, Backenhorn K, Sydenham T, Syrjakoski K, Schleutker J, et al. Nordic collaborative study of the BARD1 Cys557Ser allele in 3956 patients with cancer: enrichment in familial BRCA1/BRCA2 mutation-negative breast cancer but not in other malignancies. J Med Genet. 2006; 43: 856-62.

28. Morris JR, Pangon L, Boutell C, Katagiri T, Keep NH, Solomon E. Genetic analysis of BRCA1 ubiquitin ligase activity and its relationship to breast cancer susceptibility. Hum Mol Genet. 2006; 15: 599-606.

29. Ding DP, Zhang Y, Ma WL, He XF, Wang W, Yu HL, et al. Lack of association between BARD1 Cys557Ser variant and breast cancer risk: a meta-analysis of 11,870 cases and 7,687 controls. J Cancer Res Clin Oncol. 2011; 137: 1463-8.

30. Shastry BS. SNPs: impact on gene function and phenotype. Methods Mol Biol. 2009; 578: 3-22. 